\title{
On the definition and properties of $p$-harmonious functions
}

\author{
JuAn J. ManfRedi, MikKo Parviainen And Julio D. Rossi
}

Abstract. We consider functions that satisfy the identity

$$
u_{\varepsilon}(x)=\frac{\alpha}{2}\left\{\sup _{\bar{B}_{\varepsilon}(x)} u_{\varepsilon}+\inf _{\bar{B}_{\varepsilon}(x)} u_{\varepsilon}\right\}+\beta f_{B_{\varepsilon}(x)} u_{\varepsilon} d y
$$

for a bounded domain in $\mathbb{R}^{n}$. Here $\varepsilon>0$ and $\alpha$, and $\beta$ are suitable nonnegative coefficients such that $\alpha+\beta=1$. In particular, we show that these functions are uniquely determined by their boundary values, approximate $p$-harmonic functions for $2 \leq p<\infty$ (for a choice of $p$ that depends on $\alpha$ and $\beta$ ), and satisfy the strong comparison principle. We also analyze their relation to the theory of tug-of-war games with noise.

Mathematics Subject Classification (2010): 91A15 (primary); 35B50, 35J25, 35J70, 49N70, 91A24 (secondary).

\section{Introduction}

The fundamental works of Doob, Hunt, Kakutani, Kolmogorov and many others have shown the profound and powerful connection between the classical linear potential theory and the corresponding probability theory. The idea behind the classical interplay is that harmonic functions and martingales share a common origin in mean value properties. This approach turns out to be useful in the nonlinear theory as well. The goal of this paper is to study functions that satisfy a nonlinear generalization of the mean value theorem

$$
u_{\varepsilon}(x)=\frac{\alpha}{2}\left\{\sup _{\bar{B}_{\varepsilon}(x)} u_{\varepsilon}+\inf _{\bar{B}_{\varepsilon}(x)} u_{\varepsilon}\right\}+\beta f_{B_{\varepsilon}(x)} u_{\varepsilon} d y,
$$

JM partially supported by NSF award DMS-1001179.

MP partially supported by The Emil Aaltonen Foundation, The Fulbright Center, and The Magnus Ehrnrooth Foundation.

JDR partially supported by project MTM2004-02223, MEC, Spain, by UBA X066 and by CONICET, Argentina.

Received May 15, 2010; accepted November 25, 2010. 
with fixed $\varepsilon>0$ and suitable nonnegative $\alpha$ and $\beta$, with $\alpha+\beta=1$.

When $u$ is harmonic, then it satisfies the well known mean value property

$$
u(x)=f_{B_{\varepsilon}(x)} u d y,
$$

that is (1.1) with $\alpha=0$ and $\beta=1$. On the other hand, functions satisfying (1.1) with $\alpha=1$ and $\beta=0$

$$
u_{\varepsilon}(x)=\frac{1}{2}\left\{\sup _{\bar{B}_{\varepsilon}(x)} u_{\varepsilon}+\inf _{\bar{B}_{\varepsilon}(x)} u_{\varepsilon}\right\}
$$

are called harmonious functions in [6] and [7]. As $\varepsilon$ goes to zero, they approximate solutions to the infinity Laplacian. To be more precise, Le Gruyer proved in [6] (see also [15]) that a uniform limit when $\varepsilon \rightarrow 0$ of a sequence of harmonious functions is a solution to $\Delta_{\infty} u=0$, where $\Delta_{\infty} u=|\nabla u|^{-2} \sum_{i j} u_{x_{i}} u_{x_{i} x_{j}} u_{x_{j}}=$ $|\nabla u|^{-2}\left\langle D^{2} u \nabla u, \nabla u\right\rangle$ is the 1-homogeneous infinity Laplacian.

Recall that the $p$-Laplacian is given by

$$
\Delta_{p} u=\operatorname{div}\left(|\nabla u|^{p-2} \nabla u\right)=|\nabla u|^{p-2}\left\{(p-2) \Delta_{\infty} u+\Delta u\right\} .
$$

Since the $p$-Laplace operator can be written as a combination of the Laplace operator and the infinity Laplacian, it seems reasonable to expect that the combination (1.1) of the averages in (1.2) and (1.3) give an approximation to a solution to the $p$ Laplacian. We will show that this is indeed the case. To be more precise, we prove that $p$-harmonious functions are uniquely determined by their boundary values and that they converge uniformly to the $p$-harmonic function with the given boundary data. Furthermore, we show that $p$-harmonious functions satisfy the strong maximum and comparison principles. Observe that the validity of the strong comparison principle is an open problem for the solutions of the $p$-Laplace equation in $\mathbb{R}^{n}$, $n \geq 3$.

Consider a two-player zero-sum stochastic game in a domain $\Omega$ described as follows: fix the step size $\varepsilon>0$ and an initial position $x_{0} \in \Omega$. The players toss a biased coin with probabilities $\alpha$ and $\beta, \alpha+\beta=1$. If the result is heads (probability $\alpha$ ), they play a tug-of-war step ([15]) as follows: a fair coin is tossed and the winner of the toss is allowed to move the game position to any $x_{1} \in \bar{B}_{\varepsilon}\left(x_{0}\right)$; while, if the result is tails (probability $\beta$ ), the game state moves to a random point in the ball $B_{\varepsilon}\left(x_{0}\right)$. They continue playing until the game position leaves the domain $\Omega$. At this time Player II pays Player I the amount determined by a pay-off function defined outside $\Omega$. Naturally, Player I tries to maximize the payoff while Player II tries to minimize it. Equation (1.1) describes the expected payoff of the above game. Intuitively, the expected payoff at the point can be calculated by considering the three cases: Player I moves, Player II moves, or a random point is chosen, with their corresponding probabilities. For a more detailed description of the game we refer to Section 2. 
In this variant of the original tug-of-war with noise formulation of Peres and Sheffield in [16] the noise is distributed uniformly on $B_{\varepsilon}(x)$. This approach allows us to use the dynamic programming principle in the form (2.3) to conclude that our game has a value and that the value is $p$-harmonious. There are indications, see Barles-Souganidis [3] and Oberman [14], that our results based on the mean value approach are likely to be useful in applications for example to numerical methods as well as in problems of analysis, cf. Armstrong-Smart [1,2] (note that this last reference does not use tools from probability theory). For a deterministic-controlbased approach see [9] and [10]. On the other hand, one can look at (1.1) as a nonlocal problem analogous to the local $p$-Laplacian. For a nonlocal version of the evolution problem with the $p$-Laplacian we refer to [8].

\subsection{Main results}

To begin with, we recall a heuristic argument from [11] to gain insight on (1.1). It follows from expansion (1.4) that $u$ is a solution to $\Delta_{p} u=0$ if and only if

$$
(p-2) \Delta_{\infty} u+\Delta u=0
$$

because this equivalence can be justified in the viscosity sense even when $\nabla u=0$ as shown in [5]. Averaging the classical Taylor expansion

$$
u(y)=u(x)+\nabla u(x) \cdot(y-x)+\frac{1}{2}\left\langle D^{2} u(x)(y-x),(y-x)\right\rangle+O\left(|y-x|^{3}\right),
$$

over $B_{\varepsilon}(x)$, we obtain

$$
u(x)-f_{B_{\varepsilon}(x)} u d y=-\frac{\varepsilon^{2}}{2(n+2)} \Delta u(x)+O\left(\varepsilon^{3}\right),
$$

when $u$ is smooth. Here we used the shorthand notation

$$
f_{B_{\varepsilon}(x)} u d y=\frac{1}{\left|B_{\varepsilon}(x)\right|} \int_{B_{\varepsilon}(x)} u d y .
$$

Then observe that gradient direction is almost the maximizing direction. Thus, summing up the two Taylor expansions roughly gives us

$$
\begin{aligned}
u(x) & -\frac{1}{2}\left\{\sup _{\bar{B}_{\varepsilon}(x)} u+\inf _{\bar{B}_{\varepsilon}(x)} u\right\} \\
& \approx u(x)-\frac{1}{2}\left\{u\left(x+\varepsilon \frac{\nabla u(x)}{|\nabla u(x)|}\right)+u\left(x-\varepsilon \frac{\nabla u(x)}{|\nabla u(x)|}\right)\right\} \\
& =-\frac{\varepsilon^{2}}{2} \Delta_{\infty} u(x)+O\left(\varepsilon^{3}\right) .
\end{aligned}
$$


Next we multiply (1.6) and (1.7) by suitable constants $\alpha$ and $\beta, \alpha+\beta=1$, and add up the formulas so that we have the operator in (1.5) on the right hand side. This process gives us the choices of the constants

$$
\alpha=\frac{p-2}{p+n}, \quad \text { and } \quad \beta=\frac{2+n}{p+n},
$$

and we deduce

$$
u(x)=\frac{\alpha}{2}\left\{\sup _{\bar{B}_{\varepsilon}(x)} u+\inf _{\bar{B}_{\varepsilon}(x)} u\right\}+\beta f_{B_{\varepsilon}(x)} u d y+O\left(\varepsilon^{3}\right)
$$

as $\varepsilon \rightarrow 0$.

Consider a bounded domain $\Omega \subset \mathbb{R}^{n}$ and fix $\varepsilon>0$. To prescribe boundary values for $p$-harmonious functions, let us denote the compact boundary strip of width $\varepsilon$ by

$$
\Gamma_{\varepsilon}=\left\{x \in \mathbb{R}^{n} \backslash \Omega: \operatorname{dist}(x, \partial \Omega) \leq \varepsilon\right\}
$$

Definition 1.1. The function $u_{\varepsilon}$ is $p$-harmonious in $\Omega$ with a bounded Borel function $F: \Gamma_{\varepsilon} \rightarrow \mathbb{R}$ as boundary values if

$$
u_{\varepsilon}(x)=\frac{\alpha}{2}\left\{\sup _{\bar{B}_{\varepsilon}(x)} u_{\varepsilon}+\inf _{\bar{B}_{\varepsilon}(x)} u_{\varepsilon}\right\}+\beta f_{B_{\varepsilon}(x)} u_{\varepsilon} d y \quad \text { for every } \quad x \in \Omega,
$$

where $\alpha, \beta$ are defined in (1.8), and

$$
u_{\varepsilon}(x)=F(x), \quad \text { for every } \quad x \in \Gamma_{\varepsilon} .
$$

The reason for using the boundary strip $\Gamma_{\varepsilon}$ instead of simply using the boundary $\partial \Omega$ is the fact that for $x \in \Omega$ the ball $\bar{B}_{\varepsilon}(x)$ is not necessarily contained in $\Omega$.

To prove our main results, we assume that $2 \leq p<\infty$. The case $p=\infty$ is considered in [6] and [15].

First, with a fixed boundary data, there exists a unique $p$-harmonious function.

Theorem 1.2. Let $\Omega \subset \mathbb{R}^{n}$ be a bounded open set. Then there exists a unique p-harmonious function in $\Omega$ with given boundary values $F$.

Furthermore, $p$-harmonious functions satisfy the strong maximum principle.

Theorem 1.3. Let $\Omega \subset \mathbb{R}^{n}$ be a bounded, open, and connected set. If $u_{\varepsilon}$ is $p$ harmonious in $\Omega$ with boundary values $F$, then

$$
\sup _{\Gamma_{\varepsilon}} F \geq \sup _{\Omega} u_{\varepsilon}
$$

Moreover, if there is a point $x_{0} \in \Omega$ such that $u_{\varepsilon}\left(x_{0}\right)=\sup _{\Gamma_{\varepsilon}} F$, then $u_{\varepsilon}$ is constant in $\Omega$. 
In addition, $p$-harmonious functions with continuous boundary values satisfy the strong comparison principle. Note that the validity of the strong comparison principle is not known for the $p$-harmonic functions in $\mathbb{R}^{n}, n \geq 3$.

Theorem 1.4. Let $\Omega \subset \mathbb{R}^{n}$ be a bounded, open and connected set, and let $u_{\varepsilon}$ and $v_{\varepsilon}$ be p-harmonious functions with continuous boundary values $F_{u} \geq F_{v}$ in $\Gamma_{\varepsilon}$. Then if there exists a point $x_{0} \in \Omega$ such that $u_{\varepsilon}\left(x_{0}\right)=v_{\varepsilon}\left(x_{0}\right)$, it follows that

$$
u_{\varepsilon}=v_{\varepsilon} \text { in } \Omega \text {, }
$$

and, moreover, the boundary values satisfy

$$
F_{u}=F_{v} \text { in } \Gamma_{\varepsilon} .
$$

To prove that $p$-harmonious functions converge to the unique solution of the Dirichlet problem for the $p$-Laplacian in $\Omega$ with fixed continuous boundary values, we assume that the bounded domain $\Omega$ satisfies the following boundary regularity condition:

Boundary Regularity Condition 1.5. There exists $\delta^{\prime}>0$ and $\mu \in(0,1)$ such that for every $\delta \in\left(0, \delta^{\prime}\right]$ and $y \in \partial \Omega$ there exists a ball

$$
B_{\mu \delta}(z) \subset B_{\delta}(y) \backslash \Omega .
$$

For example, when $\Omega$ satisfies the exterior cone condition it satisfies this requirement. This is indeed the case when $\Omega$ is a Lipschitz domain.

Theorem 1.6. Let $\Omega$ be a bounded domain satisfying Condition 1.5 and $F$ be a continuous function. Consider the unique viscosity solution u to

$$
\begin{cases}\operatorname{div}\left(|\nabla u|^{p-2} \nabla u\right)(x)=0, & x \in \Omega \\ u(x)=F(x), & x \in \partial \Omega,\end{cases}
$$

and let $u_{\varepsilon}$ be the unique p-harmonious function with boundary values $F$. Then

$$
u_{\varepsilon} \rightarrow u \quad \text { uniformly in } \bar{\Omega}
$$

as $\varepsilon \rightarrow 0$.

The above limit only depends on the values of $F$ on $\partial \Omega$, and therefore any continuous extension of $\left.F\right|_{\partial \Omega}$ to $\Gamma_{\varepsilon_{0}}$ gives the same limit.

Organization of the paper. The rest of the paper is organized as follows: In Section 2 we discuss the relation between $p$-harmonious functions and tug-of-war games, in Section 3 we prove the maximum principle and the strong comparison principle of $p$-harmonious functions, and finally in Section 4 , we prove the convergence result as $\varepsilon$ goes to zero, Theorem 1.6.

ACKNOWLEDGEMENTS. The authors would like to thank Petri Juutinen for useful comments.

Part of this work was done during a visit of JDR and MP to University of Pittsburgh. They want to thank for the friendly and stimulating atmosphere found there. 


\section{2. $p$-harmonious functions and Tug-of-War games}

In this section, we describe the connection between $p$-harmonious functions and tug-of-war games. Fix $\varepsilon>0$ and consider the following two-player zero-sum game. At the beginning, a token is placed at a point $x_{0} \in \Omega$ and the players toss a biased coin with probabilities $\alpha$ and $\beta, \alpha+\beta=1$. If they get heads (probability $\alpha$ ), they play a tug-of-war, that is, a fair coin is tossed and the winner of the toss is allowed to move the game position to any $x_{1} \in \bar{B}_{\varepsilon}\left(x_{0}\right)$. On the other hand, if they get tails (probability $\beta$ ), the game state moves according to the uniform probability to a random point in the ball $B_{\varepsilon}\left(x_{0}\right)$. Then they continue playing the same game from $x_{1}$.

This procedure yields a possibly infinite sequence of game states $x_{0}, x_{1}, \ldots$ where every $x_{k}$ is a random variable. We denote by $x_{\tau} \in \Gamma_{\varepsilon}$ the first point in $\Gamma_{\varepsilon}$ in the sequence, where $\tau$ refers to the first time we hit $\Gamma_{\varepsilon}$. The payoff is $F\left(x_{\tau}\right)$, where $F: \Gamma_{\varepsilon} \rightarrow \mathbb{R}$ is a given payoff function. Player I earns $F\left(x_{\tau}\right)$ while Player II earns $-F\left(x_{\tau}\right)$.

A history of a game up to step $k$ is a vector of the first $k+1$ game states, for example, $\left(x_{0}, x_{1}, \ldots, x_{k}\right)$. We denote a set of all histories up to step $k$ by $H_{k}$, that is, it contains all possible sequences of game states of length $k$. The set of all finite histories is denoted by

$$
H=\bigcup_{k=0}^{\infty} H_{k}
$$

A strategy $S_{\mathrm{I}}$ for Player I is a function defined on $H$ that gives the next game position

$$
S_{\mathrm{I}}\left(x_{0}, x_{1}, \ldots, x_{k}\right)=x_{k+1} \in \bar{B}_{\varepsilon}\left(x_{k}\right)
$$

given a history $h$ if Player I wins the toss. Similarly Player II plays according to a strategy $S_{\text {II }}$

Let $\Omega_{\varepsilon}=\Omega \cup \Gamma_{\varepsilon} \subset \mathbb{R}^{n}$ be equipped with the natural topology, and the $\sigma$ algebra $\mathcal{B}$ of the Borel measurable sets. The space of all game sequences

$$
H^{\infty}=\Omega_{\varepsilon} \times \Omega_{\varepsilon} \times \ldots,
$$

is a product space endowed with the product topology.

Let $\left\{\mathcal{F}_{k}\right\}_{k=0}^{\infty}, \mathcal{F}_{0} \subset \mathcal{F}_{1} \subset \ldots \subset \mathcal{F}_{\infty}$, denote the following $\sigma$-algebras: the $\sigma$-algebra $\mathcal{F}_{k}$ is generated by cylinder sets of the form

$$
A_{0} \times A_{1} \times A_{2} \times \ldots \times A_{k} \times \Omega_{\varepsilon} \times \ldots
$$

with $A_{i} \in \mathcal{B}$ and $\mathcal{F}_{\infty}$ is the $\sigma$-algebra generated by $\cup_{k=0}^{\infty} \mathcal{F}_{k}$.

For $\omega=\left(\omega_{0}, \omega_{1}, \ldots\right) \in H^{\infty}$, we define

$$
x_{k}(\omega)=\omega_{k}, \quad x_{k}: H^{\infty} \rightarrow \mathbb{R}^{n}, k=0,1, \ldots
$$


so that $x_{k}$ is an $\mathcal{F}_{k}$-measurable random variable. Let

$$
\tau(\omega)=\inf \left\{k: x_{k}(\omega) \in \Gamma_{\varepsilon}, k=0,1, \ldots\right\} .
$$

This $\tau(\omega)$ is a stopping time relative to the filtration $\left\{\mathcal{F}_{k}\right\}_{k=0}^{\infty}$.

The fixed starting point $x_{0}$ and the strategies $S_{\text {I }}$ and $S_{\text {II }}$ determine a unique probability measure $\mathbb{P}_{S_{I}, S_{I I}}^{x_{0}}$ in $H^{\infty}$ relative to the product $\sigma$-algebra $\mathcal{F}^{\infty}$. This measure is built by applying Kolmogorov's extension theorem to the initial distribution $\delta_{x_{0}}(A)$, and the family of transition probabilities

$$
\begin{aligned}
& \pi_{S_{\mathrm{I}}, S_{\mathrm{II}}}\left(x_{0}(\omega), \ldots, x_{k}(\omega), A\right)=\pi_{S_{\mathrm{I}}, S_{\mathrm{II}}}\left(\omega_{0}, \ldots, \omega_{k}, A\right) \\
& \quad=\beta \frac{\left|A \cap B_{\varepsilon}\left(\omega_{k}\right)\right|}{\left|B_{\varepsilon}\left(\omega_{k}\right)\right|}+\frac{\alpha}{2} \delta_{S_{\mathrm{I}}\left(\omega_{0}, \ldots, \omega_{k}\right)}(A)+\frac{\alpha}{2} \delta_{S_{\mathrm{II}}\left(\omega_{0}, \ldots, \omega_{k}\right)}(A) .
\end{aligned}
$$

To this end, we define probability measures inductively on finite products as

$$
\begin{aligned}
& \mu_{S_{\mathrm{I}}, S_{\mathrm{II}}}^{0, x_{0}}\left(A_{0}\right)=\delta_{x_{0}}\left(A_{0}\right), \\
& \mu_{S_{\mathrm{I}}, S_{\mathrm{II}}}^{k, x_{0}}\left(A_{0} \times A_{1} \times \ldots \times A_{k-1} \times A_{k}\right) \\
& =\int_{A_{0} \times A_{1} \times \ldots \times A_{k-1}} \pi_{S_{\mathrm{I}}, S_{\mathrm{II}}}\left(\omega_{0}, \ldots, \omega_{k-1}, A_{k}\right) d \mu_{S_{\mathrm{I}}, S_{\mathrm{II}}}^{k-1, x_{0}}\left(\omega_{0}, \ldots, \omega_{k-1}\right) .
\end{aligned}
$$

Note that

$$
\mu_{S_{\mathrm{I}}, S_{\mathrm{II}}}^{k, x_{0}}\left(A_{0} \times A_{1} \times \ldots \times A_{k}\right)=\mu_{S_{\mathrm{I}}, S_{\mathrm{II}}}^{k+1, x_{0}}\left(A_{0} \times A_{1} \times \ldots \times A_{k} \times \Omega_{\varepsilon}\right) .
$$

We only concentrate on the nontrivial case $x_{0} \in A_{0}$. The first two measures are

$$
\begin{aligned}
\mu_{S_{\mathrm{I}}, S_{\mathrm{II}}}^{1, x_{0}}\left(A_{0} \times A_{1}\right) & =\int_{A_{0} \times A_{1}} d \mu_{S_{\mathrm{I}}, S_{\mathrm{II}}}^{1, x_{0}}\left(\omega_{0}, \omega_{1}\right) \\
& =\int_{A_{1}} d \mu_{S_{\mathrm{I}}, S_{\mathrm{II}}}^{1, x_{0}}\left(x_{0}, \omega_{1}\right)=\pi_{S_{\mathrm{I}}, S_{\mathrm{II}}}\left(x_{0}, A_{1}\right)
\end{aligned}
$$

and

$$
\mu_{S_{\mathrm{I}}, S_{\mathrm{II}}}^{2, x_{0}}\left(A_{0} \times A_{1} \times A_{2}\right)=\int_{A_{1}} \pi_{S_{\mathrm{I}}, S_{\mathrm{II}}}\left(x_{0}, \omega_{1}, A_{2}\right) d \pi_{S_{\mathrm{I}}, S_{\mathrm{II}}}\left(x_{0}, \omega_{1}\right) .
$$

The expected payoff, when starting from $x_{0}$ and using the strategies $S_{\mathrm{I}}, S_{\mathrm{II}}$, is

$$
\mathbb{E}_{S_{\mathrm{I}}, S_{\mathrm{II}}}^{x_{x_{0}}}\left[F\left(x_{\tau}\right)\right]=\int_{H^{\infty}} F\left(x_{\tau}(\omega)\right) d \mathbb{P}_{S_{\mathrm{I}}, S_{\mathrm{II}}}^{x_{0}}(\omega) .
$$

Note that, due to the fact that $\beta>0$, or equivalently $p<\infty$, the game ends almost surely

$$
\mathbb{P}_{S_{\mathrm{I}}, S_{\mathrm{II}}}^{x_{\mathrm{II}}}\left(\left\{\omega \in H^{\infty}: \tau(\omega)<\infty\right\}\right)=1
$$

for any choice of strategies. 
The value of the game for Player I is given by

$$
u_{\mathrm{I}}^{\varepsilon}\left(x_{0}\right)=\sup _{S_{\mathrm{I}}} \inf _{S_{\mathrm{II}}} \mathbb{E}_{S_{\mathrm{I}}, S_{\mathrm{II}}}^{x_{0}}\left[F\left(x_{\tau}\right)\right]
$$

while the value of the game for Player II is given by

$$
u_{\mathrm{II}}^{\varepsilon}\left(x_{0}\right)=\inf _{S_{\mathrm{II}}} \sup _{S_{\mathrm{I}}} \mathbb{E}_{S_{\mathrm{I}}, S_{\mathrm{II}}}^{x_{0}}\left[F\left(x_{\tau}\right)\right]
$$

The values $u_{\mathrm{I}}^{\varepsilon}\left(x_{0}\right)$ and $u_{\mathrm{II}}^{\varepsilon}\left(x_{0}\right)$ are the best expected outcomes each player can guarantee when the game starts at $x_{0}$.

We start by the statement of the Dynamic Programming Principle (DPP) applied to our game. Given the transition probabilities (2.1) we obtain

Lemma 2.1 (DPP). The value function for Player I satisfies

$$
\begin{aligned}
& u_{I}^{\varepsilon}\left(x_{0}\right)=\frac{\alpha}{2}\left\{\sup _{\bar{B}_{\varepsilon}\left(x_{0}\right)} u_{I}^{\varepsilon}+\inf _{\bar{B}_{\varepsilon}\left(x_{0}\right)} u_{I}^{\varepsilon}\right\}+\beta f_{B_{\varepsilon}\left(x_{0}\right)} u_{I}^{\varepsilon} d y, \quad x_{0} \in \Omega, \\
& u_{I}^{\varepsilon}\left(x_{0}\right)=F\left(x_{0}\right), \quad x_{0} \in \Gamma_{\varepsilon} .
\end{aligned}
$$

The value function for Player II, $u_{I I}^{\varepsilon}$, satisfies the same equation.

Formulas similar to (2.3) can be found in [13, Chapter 7]. A detailed proof adapted to our case can also be found in [12].

Let us explain intuitively why the DPP holds by considering the expectation of the payoff at $x_{0}$. Whenever the players get heads (probability $\alpha$ ) in the first coin toss, they toss a fair coin and play the tug-of-war. If Player I wins the fair coin toss in the tug-of-war (probability 1/2), she steps to a point maximizing the expectation and if Player II wins, he steps to a point minimizing the expectation. Whenever they get tails (probability $\beta$ ) in the first coin toss, the game state moves to a random point according to a uniform probability on $B_{\varepsilon}\left(x_{0}\right)$. The expectation at $x_{0}$ can be obtained by summing up these different alternatives.

We warn the reader that, in general, the value functions are discontinuous as the next example shows.

Example 2.2. Consider the case $\Omega=(0,1)$ and

$$
F(x)=u_{\mathrm{I}}^{\varepsilon}(x)= \begin{cases}1, & x \geq 1 \\ 0, & x \leq 0\end{cases}
$$

In this case the optimal strategies for both players are clear: Player I moves $\varepsilon$ to the right and Player II moves $\varepsilon$ to the left. Now, there is a positive probability of reaching $x \geq 1$ that can be uniformly bounded from below in $(0,1)$ by $C=$ $(2 / \alpha)^{-(1 / \varepsilon+1)}$. This can be seen by considering the probability of Player I winning all the time until the game ends with $x \geq 1$. Therefore $u_{\mathrm{I}}^{\varepsilon}>C>0$ in the whole 
$(0,1)$. This implies a discontinuity at $x=0$ and hence a discontinuity at $x=\varepsilon$. Indeed, first, note that $u_{\varepsilon}$ is nondecreasing and hence

$$
u_{\mathrm{I}}^{\varepsilon}(\varepsilon-)=\lim _{x \supset \varepsilon} \frac{\alpha}{2} \sup _{|x-y| \leq \varepsilon} u_{\mathrm{I}}^{\varepsilon}(y)+\frac{\beta}{2 \varepsilon} \int_{0}^{2 \varepsilon} u_{\mathrm{I}}^{\varepsilon} d y=\frac{\alpha}{2} u_{\mathrm{I}}^{\varepsilon}(2 \varepsilon-)+\frac{\beta}{2 \varepsilon} \int_{0}^{2 \varepsilon} u_{\mathrm{I}}^{\varepsilon} d y,
$$

because $\sup _{|x-y| \leq \varepsilon} u_{\mathrm{I}}^{\varepsilon}(y)=u_{\mathrm{I}}^{\varepsilon}(x+\varepsilon)$ and $\inf _{|x-\varepsilon| \leq \varepsilon} u_{\mathrm{I}}^{\varepsilon}$ is zero for $x \in(0, \varepsilon)$. However,

$$
u_{\mathrm{I}}^{\varepsilon}(\varepsilon+) \geq \frac{\alpha}{2} C+\lim _{x \searrow \varepsilon} \frac{\alpha}{2} \sup _{|x-y| \leq \varepsilon} u_{\mathrm{I}}^{\varepsilon}(y)+\frac{\beta}{2 \varepsilon} \int_{0}^{2 \varepsilon} u_{\mathrm{I}}^{\varepsilon} d y \geq \frac{\alpha}{2} C+u_{\mathrm{I}}^{\varepsilon}(\varepsilon-)
$$

because $\sup _{|x-y| \leq \varepsilon} u_{\mathrm{I}}^{\varepsilon}(y)=u_{\mathrm{I}}^{\varepsilon}(x+\varepsilon) \geq u_{\mathrm{I}}^{\varepsilon}(2 \varepsilon-)$ and $\inf _{|x-\varepsilon| \leq \varepsilon} u_{\mathrm{I}}^{\varepsilon} \geq C$ for $x>\varepsilon$.

By adapting the martingale methods used in [15], we prove a comparison principle. This also implies that $u_{\mathrm{I}}^{\varepsilon}$ and $u_{\mathrm{II}}^{\varepsilon}$ are respectively the smallest and the largest $p$-harmonious function.

Theorem 2.3. Let $\Omega \subset \mathbb{R}^{n}$ be a bounded open set. If $v$ is a p-harmonious function with boundary values $F_{v}$ in $\Gamma_{\varepsilon}$ such that $F_{v} \geq F_{u_{I}^{\varepsilon}}$, then $v \geq u_{I}^{\varepsilon}$.

Proof. We show that by choosing a strategy according to the minimal values of $v$, Player II can make the process a supermartingale. The optional stopping theorem then implies that the expectation of the process under this strategy is bounded by $v$. Moreover, this process provides an upper bound for $u_{\mathrm{I}}^{\varepsilon}$.

Player I follows any strategy and Player II follows a strategy $S_{\mathrm{II}}^{0}$ such that at $x_{k-1} \in \Omega$ he chooses to step to a point that almost minimizes $v$, that is, to a point $x_{k} \in \bar{B}_{\varepsilon}\left(x_{k-1}\right)$ such that

$$
v\left(x_{k}\right) \leq \inf _{\bar{B}_{\varepsilon}\left(x_{k-1}\right)} v+\eta 2^{-k}
$$

for some fixed $\eta>0$. We start from the point $x_{0}$. It follows that

$$
\begin{aligned}
& \mathbb{E}_{S_{\mathrm{I}}, S_{\mathrm{II}}^{0}}^{x_{0}}\left[v\left(x_{k}\right)+\eta 2^{-k} \mid x_{0}, \ldots, x_{k-1}\right] \\
& \leq \frac{\alpha}{2}\left\{\inf _{\bar{B}_{\varepsilon}\left(x_{k-1}\right)} v+\eta 2^{-k}+\sup _{\bar{B}_{\varepsilon}\left(x_{k-1}\right)} v\right\}+\beta f_{B_{\varepsilon}\left(x_{k-1}\right)} v d y+\eta 2^{-k} \\
& \leq v\left(x_{k-1}\right)+\eta 2^{-(k-1)}
\end{aligned}
$$

where we have estimated the strategy of Player I by sup and used the fact that $v$ is $p$-harmonious. Thus

$$
M_{k}=v\left(x_{k}\right)+\eta 2^{-k}
$$


is a supermartingale. Since $F_{v} \geq F_{u_{\mathrm{I}}^{\varepsilon}}$ at $\Gamma_{\varepsilon}$, we deduce

$$
\begin{aligned}
u_{\mathrm{I}}^{\varepsilon}\left(x_{0}\right) & =\sup _{S_{\mathrm{I}}} \inf _{S_{\mathrm{II}}} \mathbb{E}_{S_{\mathrm{I}}, S_{\mathrm{II}}}^{x_{0}}\left[F_{u_{\mathrm{I}}^{\varepsilon}}\left(x_{\tau}\right)\right] \leq \sup _{S_{\mathrm{I}}} \mathbb{E}_{S_{\mathrm{I}}, S_{\mathrm{II}}^{0}}^{x_{0}}\left[F_{v}\left(x_{\tau}\right)+\eta 2^{-\tau}\right] \\
& \leq \sup _{S_{\mathrm{I}}} \liminf _{k \rightarrow \infty} \mathbb{E}_{S_{\mathrm{I}}, S_{\mathrm{II}}^{0}}^{x_{0}}\left[v\left(x_{\tau \wedge k}\right)+\eta 2^{-(\tau \wedge k)}\right] \\
& \leq \sup _{S_{\mathrm{I}}} \mathbb{E}_{S_{\mathrm{I}}, S_{\mathrm{II}}^{0}}^{x_{0}}\left[M_{0}\right]=v\left(x_{0}\right)+\eta,
\end{aligned}
$$

where $\tau \wedge k=\min (\tau, k)$, and we used Fatou's lemma as well as the optional stopping theorem for $M_{k}$. Since $\eta$ was arbitrary this proves the claim.

Similarly, we can prove that $u_{\mathrm{II}}^{\varepsilon}$ is the largest $p$-harmonious function: Player II follows any strategy and Player I always chooses to step to the point where $v$ is almost maximized. This implies that $v\left(x_{k}\right)-\eta 2^{-k}$ is a submartingale. Fatou's lemma and the optional stopping theorem then prove the claim.

Next we show that the game has a value. This together with the previous comparison principle proves the uniqueness of $p$-harmonious functions with given boundary values.

Theorem 2.4. Let $\Omega \subset \mathbb{R}^{n}$ be a bounded open set, and $F$ a given boundary data in $\Gamma_{\varepsilon}$. Then $u_{I}^{\varepsilon}=u_{I I}^{\varepsilon}$, that is, the game has a value.

Proof. Clearly, $u_{\mathrm{I}}^{\varepsilon} \leq u_{\mathrm{II}}^{\varepsilon}$ always holds, so we are left with the task of showing that $u_{\mathrm{II}}^{\varepsilon} \leq u_{\mathrm{I}}^{\varepsilon}$. To see this we use the same method as in the proof of the previous theorem: Player II follows a strategy $S_{\mathrm{II}}^{0}$ such that at $x_{k-1} \in \Omega$, he always chooses to step to a point that almost minimizes $u_{\mathrm{I}}^{\varepsilon}$, that is, to a point $x_{k}$ such that

$$
u_{\mathrm{I}}^{\varepsilon}\left(x_{k}\right) \leq \inf _{\bar{B}_{\varepsilon}\left(x_{k-1}\right)} u_{\mathrm{I}}^{\varepsilon}+\eta 2^{-k},
$$

for a fixed $\eta>0$. We start from the point $x_{0}$. It follows that from the choice of strategies and the dynamic programming principle for $u_{\mathrm{I}}^{\varepsilon}$ that

$$
\begin{aligned}
\mathbb{E}_{S_{\mathrm{I}}, S_{\mathrm{II}}^{0}}^{x_{0}} & {\left[u_{\mathrm{I}}^{\varepsilon}\left(x_{k}\right)+\eta 2^{-k} \mid x_{0}, \ldots, x_{k-1}\right] } \\
& \leq \frac{\alpha}{2}\left\{\sup _{\bar{B}_{\varepsilon}\left(x_{k-1}\right)} u_{\mathrm{I}}^{\varepsilon}+\inf _{\bar{B}_{\varepsilon}\left(x_{k-1}\right)} u_{\mathrm{I}}^{\varepsilon}+\eta 2^{-k}\right\}+\beta f_{B_{\varepsilon}\left(x_{k-1}\right)} u_{\mathrm{I}}^{\varepsilon} d y+\eta 2^{-k} \\
& =u_{\mathrm{I}}^{\varepsilon}\left(x_{k-1}\right)+\eta 2^{-(k-1)} .
\end{aligned}
$$

Thus

$$
M_{k}=u_{\mathrm{I}}^{\varepsilon}\left(x_{k}\right)+\eta 2^{-k}
$$


is a supermartingale. We get by Fatou's lemma and the optional stopping theorem that

$$
\begin{aligned}
u_{\mathrm{II}}^{\varepsilon}\left(x_{0}\right) & =\inf _{S_{\mathrm{II}}} \sup _{S_{\mathrm{I}}} \mathbb{E}_{S_{\mathrm{I}}, S_{\mathrm{II}}}^{x_{0}}\left[F\left(x_{\tau}\right)\right] \leq \sup _{S_{\mathrm{I}}} \mathbb{E}_{S_{\mathrm{I}}, S_{\mathrm{II}}^{0}}^{x_{0}}\left[F\left(x_{\tau}\right)+\eta 2^{-\tau}\right] \\
& \leq \sup _{S_{\mathrm{I}}} \liminf _{k \rightarrow \infty} \mathbb{E}_{S_{\mathrm{I}}, S_{\mathrm{II}}^{0}}^{x_{0}}\left[u_{\mathrm{I}}^{\varepsilon}\left(x_{\tau \wedge k}\right)+\eta 2^{-(\tau \wedge k)}\right] \\
& \leq \sup _{S_{\mathrm{I}}} \mathbb{E}_{S_{\mathrm{I}}, S_{\mathrm{II}}^{0}}^{x_{0}}\left[u_{\mathrm{I}}^{\varepsilon}\left(x_{0}\right)+\eta\right]=u_{\mathrm{I}}^{\varepsilon}\left(x_{0}\right)+\eta .
\end{aligned}
$$

Similarly to the previous theorem, we also used the fact that the game ends almost surely. Since $\eta>0$ is arbitrary, this completes the proof.

Theorems 2.3 and 2.4 imply Theorem 1.2.

Proof of Theorem 1.2. Due to the dynamic programming principle, the values of the games are $p$-harmonious functions. This proves the existence part of Theorem 1.2. Theorems 2.3 and 2.4 imply the uniqueness part of Theorem 1.2.

Corollary 2.5. The value of the game with pay-off function $F$ coincides with the p-harmonious function with boundary values $F$.

\section{Maximum principles for $p$-harmonious functions}

In this section, we show that the strong maximum and strong comparison principles hold for $p$-harmonious functions. The latter result is interesting since the strong comparison principle is not known for $p$-harmonic functions in $\mathbb{R}^{n}$ for $n \geq 3$.

We start with the strong maximum principle: The $p$-harmonious function $u_{\varepsilon}$ attains its maximum at the boundary. Furthermore, if this value is also attained inside the domain, then $u_{\varepsilon}$ is constant.

Proof of Theorem 1.3. The proof uses the fact that if the maximum is attained inside the domain then all the quantities in the definition of a $p$-harmonious function must be equal to the maximum. This is possible in a connected domain only if the function is constant.

In Section 4 below, we will prove (see Lemma 4.3) that a $p$-harmonious function $u_{\varepsilon}$ with a boundary data $F$ satisfies

$$
\sup _{\Omega}\left|u_{\varepsilon}\right| \leq \sup _{\Gamma_{\varepsilon}}|F|
$$

Assume then that there exists a point $x_{0} \in \Omega$ such that

$$
u_{\varepsilon}\left(x_{0}\right)=\sup _{\Omega} u_{\varepsilon}=\sup _{\Gamma_{\varepsilon}} F \text {. }
$$


Then we employ the definition of a $p$-harmonious function, Definition 1.1, and obtain

$$
u_{\varepsilon}\left(x_{0}\right)=\frac{\alpha}{2}\left\{\sup _{\bar{B}_{\varepsilon}\left(x_{0}\right)} u_{\varepsilon}+\inf _{\bar{B}_{\varepsilon}\left(x_{0}\right)} u_{\varepsilon}\right\}+\beta f_{B_{\varepsilon}\left(x_{0}\right)} u_{\varepsilon} d y .
$$

Since $u_{\varepsilon}\left(x_{0}\right)$ is the maximum, the terms

$$
\sup _{\bar{B}_{\varepsilon}\left(x_{0}\right)} u_{\varepsilon}, \quad \inf _{\bar{B}_{\varepsilon}\left(x_{0}\right)} u_{\varepsilon}, \quad \text { and } \quad f_{B_{\varepsilon}\left(x_{0}\right)} u_{\varepsilon} d y
$$

on the right hand side must be smaller than or equal to $u_{\varepsilon}\left(x_{0}\right)$. On the other hand, when $p>2$, it follows that $\alpha, \beta>0$ and thus the terms must equal to $u_{\varepsilon}\left(x_{0}\right)$. Therefore,

$$
u_{\varepsilon}(x)=u_{\varepsilon}\left(x_{0}\right)=\sup _{\Omega} u_{\varepsilon}
$$

for every $x \in B_{\varepsilon}\left(x_{0}\right)$ when $p>2$. Now we can repeat the argument for each $x \in B_{\varepsilon}\left(x_{0}\right)$ and by continuing in this way, we can extend the result to the whole domain because $\Omega$ is connected. This implies that $u$ is constant everywhere when $p>2$.

Finally, if $p=2$, then (3.1) holds for almost every $x \in B_{\varepsilon}\left(x_{0}\right)$ and consequently for almost every $x$ in the whole domain. Then since

$$
u_{\varepsilon}(x)=f_{B_{\varepsilon}(x)} u_{\varepsilon} d y
$$

holds at every point in $\Omega$ and $u_{\varepsilon}$ is constant almost everywhere, it follows that $u_{\varepsilon}$ is constant everywhere.

Using similar ideas we prove the strong comparison principle: Let $u_{\varepsilon}$ and $v_{\varepsilon}$ be $p$-harmonious with boundary data $F_{u} \geq F_{v}$ in $\Gamma_{\varepsilon}$. Then if there exists a point $x_{0} \in \Omega$ with $u_{\varepsilon}\left(x_{0}\right)=v_{\varepsilon}\left(x_{0}\right)$, it follows that

$$
u_{\varepsilon}=v_{\varepsilon} \quad \text { in } \Omega
$$

and, moreover, the boundary values satisfy

$$
F_{u}=F_{v} \text { in } \Gamma_{\varepsilon} .
$$

The proof heavily uses the fact that $p<\infty$. Note that it is known that the strong comparison principle does not hold for infinity harmonic functions.

Proof of Theorem 1.4. According to Corollary 2.5 and Theorem 2.3, $F_{u} \geq F_{v}$ implies $u_{\varepsilon} \geq v_{\varepsilon}$. By the definition of a $p$-harmonious function, we have

$$
u_{\varepsilon}\left(x_{0}\right)=\frac{\alpha}{2}\left\{\sup _{\bar{B}_{\varepsilon}\left(x_{0}\right)} u_{\varepsilon}+\inf _{\bar{B}_{\varepsilon}\left(x_{0}\right)} u_{\varepsilon}\right\}+\beta f_{B_{\varepsilon}\left(x_{0}\right)} u_{\varepsilon} d y
$$


and

$$
v_{\varepsilon}\left(x_{0}\right)=\frac{\alpha}{2}\left\{\sup _{\bar{B}_{\varepsilon}\left(x_{0}\right)} v_{\varepsilon}+\inf _{\bar{B}_{\varepsilon}\left(x_{0}\right)} v_{\varepsilon}\right\}+\beta f_{B_{\varepsilon}\left(x_{0}\right)} v_{\varepsilon} d y .
$$

Next we compare the right hand sides. Because $u_{\varepsilon} \geq v_{\varepsilon}$, it follows that

$$
\begin{aligned}
\sup _{\bar{B}_{\varepsilon}\left(x_{0}\right)} u_{\varepsilon} & \leq \sup _{\bar{B}_{\varepsilon}\left(x_{0}\right)} v_{\varepsilon}, \\
\inf u_{\varepsilon} & \leq \inf _{\bar{B}_{\varepsilon}\left(x_{0}\right)} v_{\varepsilon}, \quad \text { and } \\
\bar{B}_{\varepsilon}\left(x_{0}\right) & \\
f_{B_{\varepsilon}\left(x_{0}\right)} u_{\varepsilon} d y & \leq f_{B_{\varepsilon}\left(x_{0}\right)} v_{\varepsilon} d y .
\end{aligned}
$$

Since

$$
u_{\varepsilon}\left(x_{0}\right)=v_{\varepsilon}\left(x_{0}\right)
$$

we must have equalities in (3.2). In particular, we have equality in the third inequality in (3.2), and thus

$$
u_{\varepsilon}=v_{\varepsilon} \quad \text { almost everywhere in } \quad B_{\varepsilon}\left(x_{0}\right) .
$$

Again, the connectedness of $\Omega$ immediately implies that

$$
u_{\varepsilon}=v_{\varepsilon} \quad \text { almost everywhere in } \quad \Omega \cup \Gamma_{\varepsilon} .
$$

In particular,

$$
F_{u}=F_{v} \quad \text { everywhere in } \quad \Gamma_{\varepsilon}
$$

since $F_{u}$ and $F_{v}$ are continuous. Because the boundary values coincide, the uniqueness result, Theorem 1.2 , shows that $u_{\varepsilon}=v_{\varepsilon}$ everywhere in $\Omega$.

\section{Convergence to the $p$-harmonic function as $\varepsilon \rightarrow 0$}

In this section, we show that $p$-harmonious functions with a fixed boundary data converge to the unique $p$-harmonic function. First, we prove a convergence result under additional assumptions by employing game theoretic arguments from [15] and [16]. Then we present a different proof that avoids the technical restrictions. The second proof uses a fact that although $p$-harmonious functions are, in general, discontinuous, they are, in a certain sense, asymptotically uniformly continuous.

Let $\Omega$ be a bounded open set. We assume below that $u$ is $p$-harmonic in an open set $\Omega^{\prime}$ such that $\Omega \cup \Gamma_{\varepsilon} \subset \Omega^{\prime}$. In addition, we assume that $\nabla u \neq 0$ in $\Omega^{\prime}$. This assumption guarantees that $u$ is real analytic according to a classical theorem of Hopf [4], and thus equation (4.1) below holds with a uniform error term in $\Omega$. Later we show how to deal directly with the Dirichlet problem without this extra assumption. 
Theorem 4.1. Let $u$ be $p$-harmonic with nonvanishing gradient $\nabla u \neq 0$ in $\Omega^{\prime}$ as above and let $u_{\varepsilon}$ be the p-harmonious function in $\Omega$ with the boundary values $u$ in $\Gamma_{\varepsilon}$. Then

$$
u_{\varepsilon} \rightarrow u \quad \text { uniformly in } \bar{\Omega}
$$

as $\varepsilon \rightarrow 0$.

Proof. The proof uses some ideas from the proof of [16, Theorem 2.4]. As stated at the end of Section 2, the $p$-harmonious function with boundary values coincides with the value of the game and thus we can use a game theoretic approach.

Recall from the introduction (see also [11]) that $u$ satisfies

$$
u(x)=\frac{\alpha}{2}\left\{\sup _{\bar{B}_{\varepsilon}(x)} u+\inf _{\bar{B}_{\varepsilon}(x)} u\right\}+\beta f_{B_{\varepsilon}(x)} u d y+O\left(\varepsilon^{3}\right)
$$

with a uniform error term for $x \in \bar{\Omega}$ as $\varepsilon \rightarrow 0$. The error term is uniform due to our assumptions on $u$.

Assume, for the moment, that $p>2$ implying $\alpha>0$ so that the strategies are relevant. Now, Player II follows a strategy $S_{\mathrm{II}}^{0}$ such that at a point $x_{k-1}$ he chooses to step to a point that minimizes $u$, that is, to a point $x_{k} \in B_{\varepsilon}\left(x_{k-1}\right)$ such that

$$
u\left(x_{k}\right)=\inf _{\bar{B}_{\varepsilon}\left(x_{k-1}\right)} u(y) .
$$

Choose $C_{1}>0$ such that $\left|O\left(\varepsilon^{3}\right)\right| \leq C_{1} \varepsilon^{3}$. Under the strategy $S_{\mathrm{II}}^{0}$

$$
M_{k}=u\left(x_{k}\right)-C_{1} k \varepsilon^{3}
$$

is a supermartingale. Indeed,

$$
\begin{aligned}
& \mathbb{E}_{S_{\mathrm{I}}, S_{\mathrm{II}}^{0}}^{x_{0}}\left(u\left(x_{k}\right)-C_{1} k \varepsilon^{3} \mid x_{0}, \ldots, x_{k-1}\right) \\
& \leq \frac{\alpha}{2}\left\{\sup _{\bar{B}_{\varepsilon}\left(x_{k-1}\right)} u+\inf _{\bar{B}_{\varepsilon}\left(x_{k-1}\right)} u\right\}+\beta f_{B_{\varepsilon}\left(x_{k-1}\right)} u d y-C_{1} k \varepsilon^{3} \\
& \leq u\left(x_{k-1}\right)-C_{1}(k-1) \varepsilon^{3} .
\end{aligned}
$$

The first inequality follows from the choice of the strategy and the second from (4.1). Now we can estimate $u_{\mathrm{II}}^{\varepsilon}\left(x_{0}\right)$ by using Fatou's lemma and the optional stop- 
ping theorem for supermartingales. We have

$$
\begin{aligned}
& u_{\mathrm{II}}^{\varepsilon}\left(x_{0}\right)=\inf _{S_{\mathrm{II}}} \sup _{S_{\mathrm{I}}} \mathbb{E}_{S_{\mathrm{I}}, S_{\mathrm{II}}}^{x_{0}}\left[F\left(x_{\tau}\right)\right] \\
& \leq \sup _{S_{\mathrm{I}}} \mathbb{E}_{S_{\mathrm{I}}, S_{\mathrm{II}}^{0}}^{x_{\mathrm{II}}}\left[u\left(x_{\tau}\right)\right] \\
& =\sup _{S_{\mathrm{I}}} \mathbb{E}_{S_{\mathrm{I}}, S_{\mathrm{II}}^{0}}^{x_{0}}\left[u\left(x_{\tau}\right)+C_{1} \tau \varepsilon^{3}-C_{1} \tau \varepsilon^{3}\right] \\
& \leq \sup _{S_{\mathrm{I}}}\left(\liminf _{k \rightarrow \infty} \mathbb{E}_{S_{\mathrm{I}}, S_{\mathrm{II}}^{x_{0}}}\left[u\left(x_{\tau \wedge k}\right)-C_{1}(\tau \wedge k) \varepsilon^{3}\right]+C_{1} \varepsilon^{3} \mathbb{E}_{S_{\mathrm{I}}, S_{\mathrm{II}}^{0}}^{x_{0}}[\tau]\right) \\
& \leq u\left(x_{0}\right)+C_{1} \varepsilon^{3} \sup _{S_{\mathrm{I}}} \mathbb{E}_{S_{\mathrm{I}}, S_{\mathrm{II}}^{0}}^{x_{0}}[\tau] .
\end{aligned}
$$

This inequality and the analogous argument for Player I implies for $u_{\varepsilon}=u_{\mathrm{II}}^{\varepsilon}=u_{I}^{\varepsilon}$ that

$$
u\left(x_{0}\right)-C_{1} \varepsilon^{3} \inf _{S_{\mathrm{II}}} \mathbb{E}_{S_{\mathrm{I}}^{0}, S_{I I}}^{x_{0}}[\tau] \leq u_{\varepsilon}\left(x_{0}\right) \leq u\left(x_{0}\right)+C_{1} \varepsilon^{3} \sup _{S_{\mathrm{I}}} \mathbb{E}_{S_{\mathrm{I}}, S_{\mathrm{II}}}^{x_{0}}[\tau] .
$$

Letting $\varepsilon \rightarrow 0$ the proof is completed if we prove that there exists $C$ such that

$$
\mathbb{E}_{S_{\mathrm{I}}, S_{\mathrm{II}}^{0}}^{x_{0}}[\tau] \leq C \varepsilon^{-2} .
$$

To establish this bound, we show that

$$
\tilde{M}_{k}=-u\left(x_{k}\right)^{2}+u\left(x_{0}\right)^{2}+C_{2} \varepsilon^{2} k
$$

is a supermartingale for small enough $\varepsilon>0$. If Player II wins the toss, we have

$$
u\left(x_{k}\right)-u\left(x_{k-1}\right) \leq-C_{3} \varepsilon
$$

because $\nabla u \neq 0$, as we can choose for example $C_{3}=\inf _{x \in \Omega}|\nabla u| / 2$. It follows that

$$
\begin{aligned}
& \mathbb{E}_{S_{\mathrm{I}}, S_{\mathrm{II}}^{0}}^{x_{0}}\left[\left(\left(u\left(x_{k}\right)-u\left(x_{k-1}\right)\right)^{2} \mid x_{0}, \ldots, x_{k-1}\right]\right. \\
& \quad \geq \frac{\alpha}{2}\left(\left(-C_{3} \varepsilon\right)^{2}+0\right)+\beta \cdot 0=\frac{\alpha C_{3}^{2}}{2} \varepsilon^{2} .
\end{aligned}
$$

We calculate

$$
\begin{aligned}
& \mathbb{E}_{S_{\mathrm{I}}, S_{\mathrm{II}}^{0}}^{x_{0}}\left[\tilde{M}_{k}-\tilde{M}_{k-1} \mid x_{0}, \ldots, x_{k-1}\right] \\
& =\mathbb{E}_{S_{\mathrm{I}}, S_{\mathrm{II}}^{0}}^{x_{0}}\left[-u\left(x_{k}\right)^{2}+u\left(x_{k-1}\right)^{2}+C_{2} \varepsilon^{2} \mid x_{0}, \ldots, x_{k-1}\right] \\
& =\mathbb{E}_{S_{\mathrm{I}}, S_{\mathrm{II}}^{0}}^{x_{0}}\left[-\left(u\left(x_{k}\right)-u\left(x_{k-1}\right)\right)^{2} \mid x_{0}, \ldots, x_{k-1}\right] \\
& \quad \quad-\mathbb{E}_{S_{\mathrm{I}}, S_{\mathrm{II}}^{0}}^{x_{0}}\left[2\left(u\left(x_{k}\right)-u\left(x_{k-1}\right)\right) u\left(x_{k-1}\right) \mid x_{0}, \ldots, x_{k-1}\right]+C_{2} \varepsilon^{2} .
\end{aligned}
$$


By subtracting a constant if necessary, we may assume that $u<0$. Moreover, $u\left(x_{k-1}\right)$ is determined by the point $x_{k-1}$, and thus, we can estimate the second term on the right hand side as

$$
\begin{aligned}
& -\mathbb{E}_{S_{\mathrm{I}}, S_{\mathrm{II}}^{0}}^{x_{0}}\left[2\left(u\left(x_{k}\right)-u\left(x_{k-1}\right)\right) u\left(x_{k-1}\right) \mid x_{0}, \ldots, x_{k-1}\right] \\
= & -2 u\left(x_{k-1}\right)\left(\mathbb{E}_{S_{\mathrm{I}}, S_{\mathrm{II}}^{0}}^{x_{0}}\left[u\left(x_{k}\right) \mid x_{0}, \ldots, x_{k-1}\right]-u\left(x_{k-1}\right)\right) \\
\leq & 2\|u\|_{\infty} C_{1} \varepsilon^{3} .
\end{aligned}
$$

The last inequality follows from (4.1) similarly as estimate (4.2). This together with (4.5) and (4.6) implies

$$
\mathbb{E}_{S_{\mathrm{I}}, S_{\mathrm{II}}^{0}}^{x_{0}}\left[\tilde{M}_{k}-\tilde{M}_{k-1} \mid x_{0}, \ldots, x_{k-1}\right] \leq 0,
$$

when

$$
-\varepsilon^{2} \alpha C_{3}^{2} / 2+2\|u\|_{\infty} C_{1} \varepsilon^{3}+C_{2} \varepsilon^{2} \leq 0 .
$$

This holds if we choose, for example, $C_{2}$ such that $C_{3} \geq 2 \sqrt{C_{2} / \alpha}$ and take $\varepsilon<$ $C_{2} /\left(2\|u\|_{\infty} C_{1}\right)$. Thus, $\tilde{M}_{k}$ is a supermartingale. Recall that we assumed that $p>2$ implying $\alpha>0$.

According to the optional stopping theorem for supermartingales

$$
\mathbb{E}_{S_{\mathrm{I}}, S_{\mathrm{II}}^{0}}^{x_{0}}\left[\tilde{M}_{\tau \wedge k}\right] \leq \tilde{M}_{0}=0,
$$

and thus

$$
C_{2} \varepsilon^{2} \mathbb{E}_{S_{\mathrm{I}}, S_{\mathrm{II}}^{0}}^{x_{0}}[\tau \wedge k] \leq \mathbb{E}_{S_{\mathrm{I}}, S_{\mathrm{II}}^{0}}^{x_{0}}\left[u\left(x_{\tau \wedge k}\right)^{2}-u\left(x_{0}\right)^{2}\right] .
$$

The result follows by passing to the limit with $k$ since $u$ is bounded in $\Omega$.

Finally, if $p=2$, then the mean value property holds without a correction for $u$ due to the classical mean value property for harmonic functions and the claim immediately follows by repeating the beginning of the proof till (4.3) without the correction term.

Above we obtained the convergence result for $p$-harmonious functions under the extra assumption that $\nabla u \neq 0$. Now we show how to deal directly with the Dirichlet problem and give a different proof for the uniform convergence without using this hypothesis. The proof is based on a variant of the classical ArzelaAscoli's compactness lemma, Lemma 4.2. The functions $u_{\varepsilon}$ are not continuous, in general, as shown in Example 2.2. Nonetheless, the jumps can be controlled and we will show that the $p$-harmonious functions are asymptotically uniformly continuous as shown in Theorem 4.6. 
Lemma 4.2. Let $\left\{u_{\varepsilon}: \bar{\Omega} \rightarrow \mathbb{R}, \varepsilon>0\right\}$ be a set of functions such that

(1) there exists $C>0$ so that $\left|u_{\varepsilon}(x)\right|<C$ for every $\varepsilon>0$ and every $x \in \bar{\Omega}$,

(2) given $\eta>0$ there are constants $r_{0}$ and $\varepsilon_{0}$ such that for every $\varepsilon<\varepsilon_{0}$ and any $x, y \in \bar{\Omega}$ with $|x-y|<r_{0}$ it holds

$$
\left|u_{\varepsilon}(x)-u_{\varepsilon}(y)\right|<\eta \text {. }
$$

Then, there exists a uniformly continuous function $u: \bar{\Omega} \rightarrow \mathbb{R}$ and a subsequence still denoted by $\left\{u_{\varepsilon}\right\}$ such that

$$
u_{\varepsilon} \rightarrow u \quad \text { uniformly in } \bar{\Omega},
$$

as $\varepsilon \rightarrow 0$.

Proof. First, we find a candidate to be the uniform limit $u$. Let $X \subset \bar{\Omega}$ be a dense countable set. Because functions are uniformly bounded, a diagonal procedure provides a subsequence still denoted by $\left\{u_{\varepsilon}\right\}$ that converges for all $x \in X$. Let $u(x)$ denote this limit. Note that at this point $u$ is defined only for $x \in X$.

By assumption, given $\eta>0$, there exists $r_{0}$ such that for any $x, y \in X$ with $|x-y|<r_{0}$ it holds

$$
|u(x)-u(y)|<\eta .
$$

Hence, we can extend $u$ to the whole $\bar{\Omega}$ continuously by setting

$$
u(z)=\lim _{X \ni x \rightarrow z} u(x) .
$$

Our next step is to prove that $\left\{u_{\varepsilon}\right\}$ converges to $u$ uniformly. We choose a finite covering

$$
\bar{\Omega} \subset \bigcup_{i=1}^{N} B_{r}\left(x_{i}\right)
$$

and $\varepsilon_{0}>0$ such that

$$
\left|u_{\varepsilon}(x)-u_{\varepsilon}\left(x_{i}\right)\right|,\left|u(x)-u\left(x_{i}\right)\right|<\eta / 3
$$

for every $x \in B_{r}\left(x_{i}\right)$ and $\varepsilon<\varepsilon_{0}$ as well as

$$
\left|u_{\varepsilon}\left(x_{i}\right)-u\left(x_{i}\right)\right|<\eta / 3,
$$

for every $x_{i}$ and $\varepsilon<\varepsilon_{0}$. To obtain the last inequality, we used the fact that $N<\infty$. Thus for any $x \in \bar{\Omega}$, we can find $x_{i}$ so that $x \in B_{r}\left(x_{i}\right)$ and

$$
\begin{aligned}
\mid u_{\varepsilon}(x) & -u(x) \mid \\
& \leq\left|u_{\varepsilon}(x)-u_{\varepsilon}\left(x_{i}\right)\right|+\left|u_{\varepsilon}\left(x_{i}\right)-u\left(x_{i}\right)\right|+\left|u\left(x_{i}\right)-u(x)\right| \\
& <\eta
\end{aligned}
$$

for every $\varepsilon<\varepsilon_{0}$, where $\varepsilon_{0}$ is independent of $x$. 
Next we show that for fixed $F$, a family of $p$-harmonious functions, with $\varepsilon$ as the parameter, satisfies the conditions of Lemma 4.2. First observe that $p$ harmonious functions are bounded since

$$
\min _{y \in \Gamma_{\varepsilon}} F(y) \leq F\left(x_{\tau}\right) \leq \max _{y \in \Gamma_{\varepsilon}} F(y)
$$

for any $x_{\tau} \in \Gamma_{\varepsilon}$ implies:

Lemma 4.3. A p-harmonious function $u_{\varepsilon}$ with boundary values $F$ satisfies

$$
\min _{y \in \Gamma_{\varepsilon}} F(y) \leq u_{\varepsilon}(x) \leq \max _{y \in \Gamma_{\varepsilon}} F(y) .
$$

Next we will show that $p$-harmonious functions are asymptotically uniformly continuous. We give two proofs for this result. The first proof applies Theorem 2.3 and a comparison with solutions for the $p$-Dirichlet problem in annular domains. We also use Theorem 4.1 for these solutions, which satisfy the conditions of the theorem. The proof utilizes some ideas from [16] but does not explicitly employ probabilistic tools.

Lemma 4.4. Let $\left\{u_{\varepsilon}\right\}$ be a family of p-harmonious functions in $\Omega$ with a fixed continuous boundary data F. Then this family satisfies condition (4.2) in Lemma 4.2.

Proof. Observe that the case $x, y \in \Gamma_{\varepsilon}$ readily follows from the continuity of $F$, and thus we can concentrate on the cases $x \in \Omega, y \in \Gamma_{\varepsilon}$, and $x, y \in \Omega$.

We divide the proof into three steps: First for $x \in \Omega, y \in \Gamma_{\varepsilon}$, we employ comparison with a $p$-harmonious function close to a solution for the $p$-Dirichlet problem in an annular domain. It follows that the $p$-harmonious function with the boundary data $F$ is bounded close to $y \in \Gamma_{\varepsilon}$ by a slightly smaller constant than the maximum of the boundary values. Second, we iterate this argument to show that the $p$-harmonious function is close to the boundary values near $y \in \Gamma_{\varepsilon}$ when $\varepsilon$ is small. Third, we extend this result to the case $x, y \in \Omega$ by translation, by taking the boundary values from the strip already controlled during the previous steps.

To start, we choose $B_{\mu \delta}(z) \subset B_{\delta}(y) \backslash \Omega, \delta<\delta^{\prime}$, by Condition 1.5, and consider a problem

$$
\begin{cases}\operatorname{div}\left(|\nabla u|^{p-2} \nabla u\right)(x)=0, & x \in B_{4 \delta}(z) \backslash \bar{B}_{\mu \delta}(z), \\ u(x)=\sup _{B_{5 \delta}(y) \cap \Gamma_{\varepsilon}} F, & x \in \partial B_{\mu \delta}(z), \\ u(x)=\sup _{\Gamma_{\varepsilon}} F, & x \in \partial B_{4 \delta}(z) .\end{cases}
$$

We denote $r=|x-z|$. This problem has an explicit, radially symmetric solution of the form

$$
u(r)=a r^{-(n-p) /(p-1)}+b
$$

when $p \neq n$ and

$$
u(r)=a \log (r)+b,
$$


when $p=n$. We extend the solutions to $B_{4 \delta+2 \varepsilon}(z) \backslash \bar{B}_{\mu \delta-2 \varepsilon}(z)$ and use the same notation for the extensions. Now because $\nabla u \neq 0$, Theorem 4.1 shows that for the $p$-harmonious functions $\left\{u_{\text {fund }}^{\varepsilon}\right\}$, which are defined as the values of the game in the set $B_{4 \delta+\varepsilon}(z) \backslash \bar{B}_{\mu \delta-\varepsilon}(z)$ with boundary values $u$, it holds that

$$
u_{\text {fund }}^{\varepsilon} \rightarrow u, \quad \text { uniformly in } \bar{B}_{4 \delta+\varepsilon}(z) \backslash B_{\mu \delta-\varepsilon}(z)
$$

as $\varepsilon \rightarrow 0$.

It follows that

$$
\left|u_{\text {fund }}^{\varepsilon}-u\right|=o(1) \quad \text { in } \quad \bar{B}_{4 \delta+\varepsilon}(z) \backslash B_{\mu \delta-\varepsilon}(z),
$$

where $o(1) \rightarrow 0$ as $\varepsilon \rightarrow 0$. For small enough $\varepsilon$, the comparison principle, Theorem 2.3, and the explicit solutions above imply that in $B_{\delta}(y) \cap \Omega \subset B_{2 \delta}(z) \cap \Omega$ there is $\theta \in(0,1)$ such that

$$
u^{\varepsilon} \leq u_{\text {fund }}^{\varepsilon}+o(1) \leq u+o(1) \leq \sup _{B_{5 \delta}(y) \cap \Gamma_{\varepsilon}} F+\theta\left(\sup _{\Gamma_{\varepsilon}} F-\sup _{B_{5 \delta}(y) \cap \Gamma_{\varepsilon}} F\right) .
$$

Observe that by writing down explicitly $a$ and $b$ in (4.9) or (4.10) above, we see that $0<\theta<1$ does not depend on $\delta$.

To prove the second step, we solve the $p$-harmonic function in $B_{\delta}(z) \backslash \bar{B}_{\mu \delta / 4}(z)$ with boundary values $\sup _{B_{5 \delta}(y) \cap \Gamma_{\varepsilon}} F$ at $\partial B_{\mu \delta / 4}(z)$ and from the previous step

$$
\sup _{B_{5 \delta}(y) \cap \Gamma_{\varepsilon}} F+\theta\left(\sup _{\Gamma_{\varepsilon}} F-\sup _{B_{5 \delta}(y) \cap \Gamma_{\varepsilon}} F\right)
$$

at $\partial B_{\delta}(z)$. Again the explicit solution and the comparison principle implies for small enough $\varepsilon>0$ that

$$
u_{\varepsilon} \leq \sup _{B_{5 \delta}(y) \cap \Gamma_{\varepsilon}} F+\theta^{2}\left(\sup _{\Gamma_{\varepsilon}} F-\sup _{B_{5 \delta}(y) \cap \Gamma_{\varepsilon}} F\right) \quad \text { in } \quad B_{\delta / 4}(y) \cap \Omega .
$$

Continuing in this way, we see that for small enough $\varepsilon>0$ that

$$
u_{\varepsilon} \leq \sup _{B_{5 \delta}(y) \cap \Gamma_{\varepsilon}} F+\theta^{k}\left(\sup _{\Gamma_{\varepsilon}} F-\sup _{B_{5 \delta}(y) \cap \Gamma_{\varepsilon}} F\right) \quad \text { in } \quad B_{\delta / 4^{k}}(y) \cap \Omega .
$$

This gives an upper bound for $u^{\varepsilon}$. The argument for the lower bound is similar. We have shown that for any $\eta>0$, we can choose small enough $\delta>0$, large enough $k$, and small enough $\varepsilon>0$ above so that for $x \in \Omega, y \in \Gamma_{\varepsilon}$ with $|x-y|<\delta / 4^{k}$ it holds

$$
\left|u_{\varepsilon}(x)-F(y)\right|<\eta .
$$

This shows that the second condition in Theorem 4.2 holds when $y \in \Gamma_{\varepsilon}$. 
Next we extend the estimate to the interior of the domain. First choose small enough $\delta$ and large enough $k$ so that

$$
\left|F\left(x^{\prime}\right)-F\left(y^{\prime}\right)\right|<\eta
$$

whenever $\left|x^{\prime}-y^{\prime}\right|<\delta / 4^{k}$, and $\varepsilon>0$ small enough so that (4.11) holds.

Next we consider a slightly smaller domain

$$
\tilde{\Omega}=\left\{z \in \Omega: \operatorname{dist}(z, \partial \Omega)>\delta / 4^{k+2}\right\}
$$

with the boundary strip

$$
\tilde{\Gamma}=\left\{z \in \bar{\Omega}: \operatorname{dist}(z, \partial \Omega) \leq \delta / 4^{k+2}\right\} .
$$

Suppose that $x, y \in \Omega$ with $|x-y|<\delta / 4^{k+2}$. First, if $x, y \in \tilde{\Gamma}$, then we can estimate

$$
\left|u_{\varepsilon}(x)-u_{\varepsilon}(y)\right| \leq 3 \eta
$$

by comparing the values at $x$ and $y$ to the nearby boundary values and using (4.11). Finally, let $x, y \in \tilde{\Omega}$ and define

$$
\tilde{F}(z)=u_{\varepsilon}(z-x+y)+3 \eta \quad \text { in } \quad \tilde{\Gamma} .
$$

We have

$$
\tilde{F}(z) \geq u_{\varepsilon}(z) \text { in } \quad \tilde{\Gamma}
$$

by (4.11), (4.12), and (4.13). Solve the $p$-harmonious function $\tilde{u}_{\varepsilon}$ in $\tilde{\Omega}$ with the boundary values $\tilde{F}$ in $\tilde{\Gamma}$. By the comparison principle and the uniqueness, we deduce

$$
u_{\varepsilon}(x) \leq \tilde{u}_{\varepsilon}(x)=u_{\varepsilon}(x-x+y)+3 \eta=u_{\varepsilon}(y)+3 \eta \quad \text { in } \quad \tilde{\Omega} .
$$

The lower bound follows by a similar argument.

The second proof for Lemma 4.4 is based on the connection to games and a choice of a strategy. In Lemma 4.6, we prove a slightly stronger estimate that implies Lemma 4.4. The proof of this lemma avoids the use of Theorem 4.1 but we assume a stronger boundary regularity condition instead.

At each step, we make a small correction in order to show that the process is a supermartingale. To show that the effect of the correction is small also in the long run, we need to estimate the expectation of the stopping time $\tau$. We bound $\tau$ by the exit time $\tau^{*}$ for a random walk in a larger annular domain with a reflecting condition on the outer boundary.

Lemma 4.5. Let us consider an annular domain $B_{R}(z) \backslash \bar{B}_{\delta}(z)$ and a random walk such that when at $x_{k-1}$, the next point $x_{k}$ is chosen according to a uniform distribution at $B_{\varepsilon}\left(x_{k-1}\right) \cap B_{R}(z)$. Let

$$
\tau^{*}=\inf \left\{k: x_{k} \in \bar{B}_{\delta}(z)\right\} .
$$


Then

$$
\mathbb{E}^{x_{0}}\left(\tau^{*}\right) \leq \frac{C(R / \delta) \operatorname{dist}\left(\partial B_{\delta}(z), x_{0}\right)+o(1)}{\varepsilon^{2}},
$$

for $x_{0} \in B_{R}(z) \backslash \bar{B}_{\delta}(z)$. Here o $(1) \rightarrow 0$ as $\varepsilon \rightarrow 0$.

Proof. We will use a solution to a corresponding Poisson problem to prove the result. Let us denote

$$
g_{\varepsilon}(x)=\mathbb{E}^{x}\left(\tau^{*}\right)
$$

The function $g_{\varepsilon}$ satisfies a dynamic programming principle

$$
g_{\varepsilon}(x)=f_{B_{\varepsilon}(x) \cap B_{R}(z)} g_{\varepsilon} d y+1
$$

because the number of steps always increases by one when making a step to one of the neighboring points at random. Further, we denote $v_{\varepsilon}(x)=\varepsilon^{2} g_{\varepsilon}(x)$ and obtain

$$
v_{\varepsilon}(x)=f_{B_{\varepsilon}(x) \cap B_{R}(z)} v_{\varepsilon} d y+\varepsilon^{2} .
$$

This formula suggests a connection to the problem

$$
\begin{cases}\Delta v(x)=-2(n+2), & x \in B_{R+\varepsilon}(z) \backslash \bar{B}_{\delta}(z), \\ v(x)=0, & x \in \partial B_{\delta}(z), \\ \frac{\partial v}{\partial v}=0, & x \in \partial B_{R+\varepsilon}(z),\end{cases}
$$

where $\frac{\partial v}{\partial v}$ refers to the normal derivative. Indeed, when $B_{\varepsilon}(x) \subset B_{R+\varepsilon}(z) \backslash \bar{B}_{\delta}(z)$, the classical calculation shows that the solution of this problem satisfies the mean value property

$$
v(x)=f_{B_{\varepsilon}(x)} v d y+\varepsilon^{2} .
$$

The solution of problem (4.15) is positive, radially symmetric, and strictly increasing in $r=|x-z|$. It takes the form $v(r)=-a r^{2}-b r^{2-n}+c$, if $n>2$ and $v(r)=-a r^{2}-b \log (r)+c$, if $n=2$.

We extend this function as a solution to the same equation to $\bar{B}_{\delta}(z) \backslash \bar{B}_{\delta-\varepsilon}(z)$ and use the same notation for the extension. Thus, $v$ satisfies (4.16) for each $B_{\varepsilon}(x) \subset B_{R+\varepsilon}(z) \backslash \bar{B}_{\delta-\varepsilon}(z)$. In addition, because $v$ is increasing in $r$, it holds for each $x \in B_{R}(z) \backslash \bar{B}_{\delta}(z)$ that

$$
f_{B_{\varepsilon}(x) \cap B_{R}(z)} v d y \leq f_{B_{\varepsilon}(x)} v d y=v(x)-\varepsilon^{2} .
$$


It follows that

$$
\mathbb{E}^{x_{0}}\left[v\left(x_{k}\right)+k \varepsilon^{2} \mid x_{0}, \ldots, x_{k-1}\right]=\int_{B_{\varepsilon}\left(x_{k-1}\right)} v d y+k \varepsilon^{2}=v\left(x_{k-1}\right)+(k-1) \varepsilon^{2},
$$

if $B_{\varepsilon}\left(x_{k-1}\right) \subset B_{R}(z) \backslash \bar{B}_{\delta-\varepsilon}(z)$, and if $B_{\varepsilon}\left(x_{k-1}\right) \backslash B_{R}(z) \neq \emptyset$, then

$$
\begin{aligned}
\mathbb{E}^{x_{0}}\left[v\left(x_{k}\right)+k \varepsilon^{2} \mid x_{0}, \ldots, x_{k-1}\right] & =\int_{B_{\varepsilon}\left(x_{k-1}\right) \cap B_{R}(z)} v d y+k \varepsilon^{2} \\
& \leq f_{B_{\varepsilon}\left(x_{k-1}\right)} v d y=v\left(x_{k-1}\right)+(k-1) \varepsilon^{2}
\end{aligned}
$$

Thus $v\left(x_{k}\right)+k \varepsilon^{2}$ is a supermartingale, and the optional stopping theorem yields

$$
\mathbb{E}^{x_{0}}\left[v\left(x_{\tau^{*} \wedge k}\right)+\left(\tau^{*} \wedge k\right) \varepsilon^{2}\right] \leq v\left(x_{0}\right)
$$

Because $x_{\tau^{*}} \in \bar{B}_{\delta}(z) \backslash \bar{B}_{\delta-\varepsilon}(z)$, we have

$$
0 \leq-\mathbb{E}^{x_{0}}\left[v\left(x_{\tau^{*}}\right)\right] \leq o(1)
$$

Furthermore, the estimate

$$
0 \leq v\left(x_{0}\right) \leq C(R / \delta) \operatorname{dist}\left(\partial B_{\delta}(z), x_{0}\right)
$$

holds for the solutions of (4.15). Thus, by passing to the limit on $k$ in (4.17), we obtain

$$
\varepsilon^{2} \mathbb{E}^{x_{0}}\left[\tau^{*}\right] \leq v\left(x_{0}\right)-\mathbb{E}\left[u\left(x_{\tau^{*}}\right)\right] \leq C(R / \delta)\left(\operatorname{dist}\left(\partial B_{\delta}(z), x_{0}\right)+o(1)\right) .
$$

This completes the proof.

Next we derive an estimate for the asymptotic uniform continuity of the family of $p$-harmonious functions which implies Lemma 4.4. For simplicity, we assume that $\Omega$ satisfies an exterior sphere condition: For each $y \in \partial \Omega$, there exists $B_{\delta}(z) \subset$ $\mathbb{R}^{n} \backslash \Omega$ such that $y \in \partial B_{\delta}(z)$. With this assumption, the iteration used in the first proof of Lemma 4.4 can be avoided. To simplify the notation and to obtain an explicit estimate, we also assume that $F$ is Lipschitz continuous in $\Gamma_{\varepsilon}$.

Lemma 4.6. Let $F$ and $\Omega$ be as above. The p-harmonious function $u_{\varepsilon}$ with the boundary data $F$ satisfies

$$
\left|u_{\varepsilon}(x)-u_{\varepsilon}(y)\right| \leq 2 \operatorname{Lip}(F) \delta+C(R / \delta)(|x-y|+o(1)),
$$

for every small enough $\delta>0$ and for every two points $x, y \in \Omega \cup \Gamma_{\varepsilon}$. 
Proof. As in the first proof of Lemma 4.4, the case $x, y \in \Gamma_{\varepsilon}$ is clear. Thus, we can concentrate on the cases $x \in \Omega$ and $y \in \Gamma_{\varepsilon}$ as well as $x, y \in \Omega$.

We utilize the connection to games. Suppose first that $x \in \Omega$ and $y \in \Gamma_{\varepsilon}$. By the exterior sphere condition, there exists $B_{\delta}(z) \subset \mathbb{R}^{n} \backslash \Omega$ such that $y \in \partial B_{\delta}(z)$. Player I chooses a strategy of pulling towards $z$, denoted by $S_{I}^{z}$. Then

$$
M_{k}=\left|x_{k}-z\right|-C \varepsilon^{2} k
$$

is a supermartingale for a constant $C$ large enough independent of $\varepsilon$. Indeed,

$$
\begin{aligned}
& \mathbb{E}_{S_{\mathrm{I}}^{z}, S_{\mathrm{II}}}^{x_{0}}\left[\left|x_{k}-z\right| \mid x_{0}, \ldots, x_{k-1}\right] \\
& \leq \frac{\alpha}{2}\left\{\left|x_{k-1}-z\right|+\varepsilon+\left|x_{k-1}-z\right|-\varepsilon\right\}+\beta f_{B_{\varepsilon}\left(x_{k-1}\right)}|x-z| d x \\
& \leq\left|x_{k-1}-z\right|+C \varepsilon^{2} .
\end{aligned}
$$

The first inequality follows from the choice of the strategy, and the second from the estimate

$$
f_{B_{\varepsilon}\left(x_{k-1}\right)}|x-z| d x \leq\left|x_{k-1}-z\right|+C \varepsilon^{2} .
$$

By the optional stopping theorem, this implies that

$$
\mathbb{E}_{S_{I}^{z}, S_{I I}}^{x_{0}}\left[\left|x_{\tau}-z\right|\right] \leq\left|x_{0}-z\right|+C \varepsilon^{2} \mathbb{E}_{S_{I}^{z}, S_{I I}}^{x_{0}}[\tau]
$$

Next we estimate $\mathbb{E}_{S_{I}^{z}, S_{I I}}^{x_{0}}[\tau]$ by the stopping time of Lemma 4.5. Player I pulls towards $z$ and Player II uses any strategy. The expectation of $\left|x_{k}-z\right|$ when at $x_{k-1}$ is at the most $\left|x_{k-1}-z\right|$ when we know that the tug-of-war occurs. On the other hand, if the random walk occurs, then we know that the expectation of $\left|x_{k}-z\right|$ is greater than or equal to $\left|x_{k-1}-z\right|$. Therefore we can bound the expectation of the original process by considering a suitable random walk in $B_{R}(z) \backslash B_{\delta}(z)$ for $B_{R}(z)$ such that $\Omega \subset B_{R / 2}(z)$. When $x_{k} \in B_{R}(z) \backslash \bar{B}_{\delta}(z)$, the successor $x_{k+1}$ is chosen according to a uniform probability in $B_{\varepsilon}(x) \cap B_{R}(z)$. The process stops when it hits $\bar{B}_{\delta}(z)$. Thus, by (4.14),

$$
\varepsilon^{2} \mathbb{E}_{S_{I}^{z}, S_{I I}}^{x_{0}}[\tau] \leq \varepsilon^{2} \mathbb{E}_{S_{I}^{z}, S_{I I}}^{x_{0}}\left[\tau^{*}\right] \leq C(R / \delta)\left(\operatorname{dist}\left(\partial B_{\delta}(z), x_{0}\right)+o(1)\right)
$$

Since $y \in \partial B_{\delta}(z)$,

$$
\operatorname{dist}\left(\partial B_{\delta}(z), x_{0}\right) \leq\left|y-x_{0}\right|,
$$


and thus, (4.19) implies

$$
\mathbb{E}_{S_{I}^{z}, S_{I I}}^{x_{0}}\left[\left|x_{\tau}-z\right|\right] \leq C(R / \delta)\left(\left|x_{0}-y\right|+o(1)\right)+\delta .
$$

We get

$$
\begin{aligned}
F(z)-C(R / \delta)(|x-y|+o(1)) & -\operatorname{Lip}(F) \delta \leq \mathbb{E}_{S_{I}^{z}, S_{I I}}^{x_{0}}\left[F\left(x_{\tau}\right)\right] \\
& \leq F(z)+C(R / \delta)(|x-y|+o(1))+\operatorname{Lip}(F) \delta .
\end{aligned}
$$

Thus, we obtain

$$
\begin{aligned}
\sup _{S_{\mathrm{I}}} \inf _{S_{\mathrm{II}}} \mathbb{E}_{S_{\mathrm{I}}, S_{\mathrm{II}}}^{x_{0}}\left[F\left(x_{\tau}\right)\right] & \geq \inf _{S_{\mathrm{II}}} \mathbb{E}_{S_{\mathrm{I}}^{z}, S_{\mathrm{II}}}^{x_{0}}\left[F\left(x_{\tau}\right)\right] \\
& \geq F(z)-C(R / \delta)\left(\left|x_{0}-y\right|+o(1)\right)-\operatorname{Lip}(F) \delta \\
& \geq F(y)-2 \operatorname{Lip}(F) \delta-C(R / \delta)\left(\left|x_{0}-y\right|+o(1)\right) .
\end{aligned}
$$

The upper bound can be obtained by choosing for Player II a strategy where he points to $z$, and thus, (4.18) follows.

We can then translate the estimate inside the domain similarly as at the end of the proof of Lemma 4.4. For completeness, we also give a probabilistic proof from [16] for this fact.

Let $x, y \in \Omega$ and fix the strategies $S_{\mathrm{I}}, S_{\mathrm{II}}$ for the game starting at $x$. We define a virtual game starting at $y$ : we use the same coin tosses and random steps as the usual game starting at $x$. Furthermore, the players adopt their strategies $S_{\mathrm{I}}^{v}, S_{\mathrm{II}}^{v}$ from the game starting at $x$, that is, when the game position is $y_{k-1}$ a player chooses the step that would be taken at $x_{k-1}$ in the game starting at $x$. We proceed in this way until for the first time $x_{k} \in \Gamma_{\varepsilon}$ or $y_{k} \in \Gamma_{\varepsilon}$. At that point we have $\left|x_{k}-y_{k}\right|=|x-y|$, and we may apply the previous steps that work for $x_{k} \in \Omega$, $y_{k} \in \Gamma_{\varepsilon}$ or for $x_{k}, y_{k} \in \Gamma_{\varepsilon}$.

Note that, thanks to Lemmas 4.3 and 4.4 (or alternatively Lemma 4.6), the family $u_{\varepsilon}$ satisfies the hypothesis of the compactness Lemma 4.2.

Corollary 4.7. Let $\left\{u_{\varepsilon}\right\}$ be a family of p-harmonious functions with a fixed continuous boundary data $F$. Then there exists a uniformly continuous $u$ and a subsequence still denoted by $\left\{u_{\varepsilon}\right\}$ such that

$$
u_{\varepsilon} \rightarrow u \quad \text { uniformly in } \bar{\Omega} \text {. }
$$

Next we prove that the limit $u$ in Corollary 4.7 is a solution to (1.9). The idea is to work in the viscosity setting and to show that the limit is a viscosity suband supersolution. To accomplish this, we utilize some ideas from [11], where $p$ harmonic functions were characterized in terms of asymptotic expansions. We start by recalling the viscosity characterization of $p$-harmonic functions, see [5]. 
Definition 4.8. For $1<p<\infty$ consider the equation

$$
-\operatorname{div}\left(|\nabla u|^{p-2} \nabla u\right)=0 .
$$

(1) A lower semi-continuous function $u$ is a viscosity supersolution if for every $\phi \in C^{2}$ such that $\phi$ touches $u$ at $x \in \Omega$ strictly from below with $\nabla \phi(x) \neq 0$, we have

$$
-(p-2) \Delta_{\infty} \phi(x)-\Delta \phi(x) \geq 0 .
$$

(2) An upper semi-continuous function $u$ is a subsolution if for every $\phi \in C^{2}$ such that $\phi$ touches $u$ at $x \in \Omega$ strictly from above with $\nabla \phi(x) \neq 0$, we have

$$
-(p-2) \Delta_{\infty} \phi(x)-\Delta \phi(x) \leq 0 .
$$

(3) Finally, $u$ is a viscosity solution if it is both a sub- and supersolution.

Theorem 4.9. Let $F$ and $\Omega$ be as in Theorem 1.6. Then the uniform limit $u$ of p-harmonious functions $\left\{u_{\varepsilon}\right\}$ is a viscosity solution to (1.9).

Proof. First, $u=F$ on $\partial \Omega$ due to Lemma 4.4, and we can focus attention on showing that $u$ is $p$-harmonic in $\Omega$ in the viscosity sense. To this end, we recall from [11] an estimate that involves the regular Laplacian $(p=2)$ and an approximation for the infinity Laplacian $(p=\infty)$. Choose a point $x \in \Omega$ and a $C^{2}$-function $\phi$ defined in a neighborhood of $x$. Let $x_{1}^{\varepsilon}$ be the point at which $\phi$ attains its minimum in $\bar{B}_{\varepsilon}(x)$

$$
\phi\left(x_{1}^{\varepsilon}\right)=\min _{y \in \bar{B}_{\varepsilon}(x)} \phi(y) .
$$

It follows from the Taylor expansions in [11] that

$$
\begin{aligned}
& \frac{\alpha}{2}\left\{\max _{y \in \bar{B}_{\varepsilon}(x)} \phi(y)+\min _{y \in \bar{B}_{\varepsilon}(x)} \phi(y)\right\}+\beta f_{B_{\varepsilon}(x)} \phi(y) d y-\phi(x) \\
& \geq \frac{\beta \varepsilon^{2}}{2(n+2)}\left((p-2)\left\langle D^{2} \phi(x)\left(\frac{x_{1}^{\varepsilon}-x}{\varepsilon}\right),\left(\frac{x_{1}^{\varepsilon}-x}{\varepsilon}\right)\right\rangle+\Delta \phi(x)\right) \\
& \quad+o\left(\varepsilon^{2}\right) .
\end{aligned}
$$

Suppose that $\phi$ touches $u$ at $x$ strictly from below and that $\nabla \phi(x) \neq 0$. Observe that according to Definition 4.8 , it is enough to test with such functions. By the uniform convergence, there exists sequence $\left\{x_{\varepsilon}\right\}$ converging to $x$ such that $u_{\varepsilon}-\phi$ has an approximate minimum at $x_{\varepsilon}$, that is, for $\eta_{\varepsilon}>0$, there exists $x_{\varepsilon}$ such that

$$
u_{\varepsilon}(x)-\phi(x) \geq u_{\varepsilon}\left(x_{\varepsilon}\right)-\phi\left(x_{\varepsilon}\right)-\eta_{\varepsilon} .
$$

Moreover, considering $\tilde{\phi}=\phi-u_{\varepsilon}\left(x_{\varepsilon}\right)-\phi\left(x_{\varepsilon}\right)$, we can assume that $\phi\left(x_{\varepsilon}\right)=u_{\varepsilon}\left(x_{\varepsilon}\right)$. Thus, by recalling the fact that $u_{\varepsilon}$ is $p$-harmonious, we obtain

$$
\eta_{\varepsilon} \geq-\phi\left(x_{\varepsilon}\right)+\frac{\alpha}{2}\left\{\max _{\bar{B}_{\varepsilon}\left(x_{\varepsilon}\right)} \phi+\min _{\bar{B}_{\varepsilon}\left(x_{\varepsilon}\right)} \phi\right\}+\beta f_{B_{\varepsilon}\left(x_{\varepsilon}\right)} \phi(y) d y,
$$


and thus, by (4.20), and choosing $\eta_{\varepsilon}=o\left(\varepsilon^{2}\right)$, we have

$$
\begin{aligned}
0 \geq & \frac{\beta \varepsilon^{2}}{2(n+2)}\left((p-2)\left\langle D^{2} \phi\left(x_{\varepsilon}\right)\left(\frac{x_{1}^{\varepsilon}-x_{\varepsilon}}{\varepsilon}\right),\left(\frac{x_{1}^{\varepsilon}-x_{\varepsilon}}{\varepsilon}\right)\right\rangle+\Delta \phi\left(x_{\varepsilon}\right)\right) \\
& +o\left(\varepsilon^{2}\right) .
\end{aligned}
$$

Since $\nabla \phi(x) \neq 0$, letting $\varepsilon \rightarrow 0$, we get

$$
0 \geq \frac{\beta}{2(n+2)}\left((p-2) \Delta_{\infty} \phi(x)+\Delta \phi(x)\right) .
$$

Therefore $u$ is a viscosity supersolution.

To prove that $u$ is a viscosity subsolution, we use a reverse inequality to (4.20) by considering the maximum point of the test function and choose a function $\phi$ that touches $u$ from above.

The above theorem gives the existence of a viscosity solution to (1.9).

End of the proof of Theorem 1.6. We just have to observe that since the viscosity solution of (1.9) is unique, then we have convergence for the whole family $\left\{u_{\varepsilon}\right\}$ as $\varepsilon \rightarrow 0$.

\section{References}

[1] S. N. ARMSTRONG and C. K. SMART, An easy proof of Jensen's theorem on the uniqueness of infinity harmonic functions, Calc. Var. Partial Differential Equations 37 (2010), 381-384.

[2] S. N. ARmstrong and C. K. SMART, A finite difference approach to the infinity Laplace equation and tug-of-war games, Trans. Amer. Math. Soc. 364 (2012), 595-636.

[3] G. BARLES and P. E. SOUGANIDIS, Convergence of approximation schemes for fully nonlinear second order equations. Asymptotic Anal. 4 (1991), 271-283.

[4] E. HopF, Über den funktionalen, insbesondere den analytischen charakter der lösungen elliptischer differentialgleichungen zweiter ordnung, Math. Z. 34 (1932), 194-233.

[5] P. JuUtinen, P. LindQVist and J. J. MANFREDI, On the equivalence of viscosity solutions and weak solutions for a quasi-linear elliptic equation, SIAM J. Math. Anal. 33 (2001), 699-717.

[6] E. LE GRUYER, On absolutely minimizing Lipschitz extensions and PDE $\Delta_{\infty}(u)=0$, NoDEA Nonlinear Differential Equations Appl. 14 (2007), 29-55.

[7] E. Le Gruyer and J. C. Archer, Harmonious extensions, SIAM J. Math. Anal. 29 (1998), 279-292.

[8] H. IshiI and NAKAmURA, A class of integral equations and approximation of p-Laplace equations, Calc. Var. Partial Differential Equations 37 (2010), 485-522.

[9] R. V. KOHN and S. SERFATY, A deterministic-control-based approach to motion by curvature, Comm. Pure Appl. Math. 59 (2006), 344-407.

[10] R. V. KOHN and S. SERFATY, A deterministic-control-based approach to fully non-linear parabolic and elliptic equations, Comm. Pure Appl. Math. 63 (2010), 1298-1350.

[11] J. J. MANFredi, M. PARVIAINEN and J. D. Rossi, An asymptotic mean value characterization of p-harmonic functions, Proc. Amer. Math. Soc. 138 (2010), 881-889. 
[12] J. J. MAnfredi, M. Parviainen and J. D. Rossi, Dynamic programming principle for tug-of-war games with noise, ESAIM Control Optim. Calc. Var. 81 (2012), 81-90.

[13] A. P. MAITRA and W. D. SudDERTH, "Discrete Gambling and Stochastic Games", Applications of Mathematics, Vol. 32, Springer-Verlag, 1996.

[14] A. M. OBERMAN, A convergent difference scheme for the infinity Laplacian: construction of absolutely minimizing Lipschitz extensions, Math. Comp. 74 (2005), 1217-1230.

[15] Y. Peres, O. SChramm, S. ShefField and D. B. Wilson, Tug-of-war and the infinity Laplacian, J. Amer. Math. Soc. 22 (2009), 167-210.

[16] Y. Peres and S. ShefField, Tug-of-war with noise: a game theoretic view of the pLaplacian, Duke Math. J. 145 (2008), 91-120.

Department of Mathematics

University of Pittsburgh

Pittsburgh, PA 15260, USA

manfredi@pitt.edu

Department of Mathematics and Statistics

P.O. Box 35

FI-40014 University of Jyväskylä

Finland

mikko.j.parviainen@jyu.fi

Departamento de Análisis Matemático

Universidad de Alicante

Ap. correos 99, 03080, Alicante, Spain

On leave from:

Departamento de Matemática

FCEyN UBA

14128, Buenos Aires, Argentina

julio.rossi@ua.es 